\title{
Time-dependent fluctuations of retention performance in an aversively motivated task
}

\author{
PASCALE GISQUET-VERRIER and TATIANA ALEXINSKY \\ Laboratoire de Physiologie Nerveuse, C.N.R.S., Gif sur Yvette, France
}

\begin{abstract}
The main aim of this series of experiments was to clearly establish the spontaneous development of retention performance following partial aversively motivated training. Experiment 1 indicated that following 15 training trials of a brightness discrimination in a Y-maze, performance spontaneously and transitorily deteriorated $1 \mathrm{~h}$ following inital training (Kamin effect) before it improved 8 to 14 days following training (long-term spontaneous improvement). Both of these fluctuations preceded the more durable deterioration that corresponds to long-term forgetting. Experiment 2 replicated the basic findings of the first experiment, always with a brightness discrimination, but using an avoidance paradigm. These results demonstrated the multiphasic nature of the retention curve and emphasized the reliability of retention performance fluctuations.
\end{abstract}

It is now well accepted that memory might be seen as consisting of a collection of attributes, each representing events or features of the training episode (see Spear, 1978; Underwood, 1969). Furthermore, it has been proposed (Spear, 1978) that these memory attributes develop over time rather independently. Thus, the retention interval must be considered as an active and dynamic phase, implicating reorganizations of the memory trace over time. Such a dynamic process implies that the retention curve does not develop monotonically over time. Indeed, besides the negatively accelerated curve of forgetting described by Ebbinghaus (1885), the literature includes numerous reports of studies exhibiting multiphasic retention functions, each obviously differing from the others inasmuch as they were obtained with different species and training tasks (Cherkin, 1971; Holloway \& Wansley, 1973; Messenger, 1971; Sanders \& Barlow, 1971). All of these curves attested that the multiphasic nature of the retention curve seems to be a general characteristic, since it is observed in various circumstances with both vertebrates and invertebrates. These results have been interpreted diversely, but always in terms of associative processes. For some authors, the retention curve revealed different memory systems-short, intermediate, and longterm (Messenger, 1971; Sanders \& Barlow, 1971). For other authors, especially for those who had demonstrated multiple retention deficits over a period of $24 \mathrm{~h}$, those fluctuations revealed periodic retrieval failures due to the biological rhythms that may induce differences in the animals' state between training and testing, that is, a statedependent type of process (Elson, Seybert, \& Ghiselli, 1977; Holloway \& Wansley, 1973; Hunsicker \& Mellgren, 1977; Wansley \& Holloway, 1975).

Two performance fluctuations have been studied carefully, but always separately. The first, the Kamin effect,

Requests for reprints should be directed to Pascale Gisquet-Verrier, LPN 2, C.N.R.S., 91190 Gif sur Yvette, France. is a well-established, U-shaped retention function due to a transitory drop in performance occurring one to several hours following incompletely learned active avoidance training in animals (Kamin, 1957). Such an effect, which has been demonstrated frequently for aversively motivated tasks, has also been described following appetitive training (for a review, see Brush, 1971; Gisquet-Verrier, 1983).

The second performance fluctuation corresponds to a spontaneous improvement of performance first demonstrated with human subjects for whom improvement occurred quickly (in the first few minutes) following initial training. This phenomenon, which was later confirmed with animals, with the best performance usually occurring several days following training, is not as well established as the Kamin effect, probably because it has not been studied to as great an extent. Moreover, the earlier studies, mainly those published prior to 1940 (A. C. Anderson, 1940; Magdsick, 1936), have been criticized because of methodological deficiencies. In addition, the authors used different terms to describe the same phenomenon observed after positively and negatively reinforced task: "reminiscence" (Bovet, Bovet-Nitti, \& Oliverio, 1969; Jaffard, Destrade, Soumireu-Mourat, \& Cardo, 1974), “incubation" (Geller \& Jarvik, 1970; Pinel \& Cooper, 1966), or simply "improvement of performance" (Gisquet-Verrier \& Alexinsky, 1986; Stanes, Brown, \& Singer, 1976). For these reasons, the existence of this fluctuation of retention performance as a real feature of memory processes has been largely ignored.

The clearest instance of long-term spontaneous improvement (LTSI) of performance may be found in the data obtained by Huppert and Deutsch (1969), who have studied the question extensively. Rats partially trained to use a brightness-discrimination to escape a shock in a Ymaze were tested for retention after intervals ranging from 30 min to 17 days. Retention performance was found to be maximal at 7 to 10 days. This result was subsequently 
confirmed in studies that used aversive situations (Price \& Cooper, 1975; Signorelli, 1976) or an appetitive situation (Stanes et al., 1976).

The principal aim of the present work was to demonstrate the multiphasicity of the retention curve and to investigate the possibility that these two transitory performance fluctuations, the Kamin effect and the LTSI, always described separately, could be obtained following the same training episode, both preceding the more durable deterioration that corresponds to long-term forgetting. Accordingly, in the first experiment, we adopted the experimental paradigm of Huppert and Deutsch (1969), who obtained a LTSI following partial brightness-discrimination escape training.

With a view to extending and generalizing these results, the aim of the second experiment was to replicate the findings of the first study using avoidance brightness discrimination, which had previously only been shown to be followed by a Kamin effect (Barrett, Leith, \& Ray, 1971; Steranka \& Barrett, 1973).

\section{EXPERIMENT 1}

\section{Method}

\section{Subjects}

One hundred and thirty naive male Sprague-Dawley rats, obtained from Iffa Credo (St. Germain sur l'Arbresle, France), served as subjects. They were 50-57 days old and weighed $250 \mathrm{~g}$ when they arrived at the laboratory. They were housed in pairs, in wire-mesh cages, and had free access to food and water.

\section{Apparatus}

Training and testing were carried out in a semi-automated black perspex Y-maze with 13-cm-wide, $60-\mathrm{cm}$-long, and 38-cm-high arms and a $25-\mathrm{cm}$ equilateral choice area. A 40-W lamp was located at the end of each arm. The floor consisted of 3-mm-diam grids spaced $9 \mathrm{~mm}$ apart. A shock generator (Campden, Model 521S), set to deliver a scrambled shock, provided the incentive for learning. Photocells, placed $42 \mathrm{~cm}$ from the entrance to each arm, were connected to an electronic card programmer that controlled the light sequence and the length of the intertrial interval. Response latency, which was recorded by stopwatch, corresponded to the time between the lighting of a new goal arm and the moment the subject broke the photobeam of this arm. The maze was housed in a darkened room adjacent to the colony room.

\section{Procedure}

The first 2 days consisted of a pretraining period during which the rats were habituated to the maze and to the experimenters. On Day 1 , the rats, in groups of 6 , were given 15 min of free exploration of the entire lighted maze. On Day 2 , individual rats were placed in the lighted maze and allowed to explore for $5 \mathrm{~min}$. The number of arms visited, which indicated the animal's level of spontaneous motor activity, was recorded. Training began the following day. All animals were given 15 training trials according to the following procedure: Each rat was placed in the lighted start arm of the Y-maze for $20 \mathrm{sec}$. The door that closed off the start arm (and which was used only during the first trial) was then opened, the initial light was turned off, and another arm was lighted. The electric shock was applied to the grid floor throughout the maze, except the terminal $30 \mathrm{~cm}$ of the lighted arm. The rat had to run to the lighted arm to escape the footshock. A response was scored as incorrect if the rat traversed a distance of at least $11 \mathrm{~cm}$ into the unlit alley before escaping into the lighted alley. Animals that failed to respond within $120 \mathrm{sec}$ were pushed manually into the lighted arm; for this, one error and a response latency of $120 \mathrm{sec}$ were recorded. The rat remained in the safe compartment for a 20 -sec period, which constituted the intertrial interval. An exit from the lighted alley led to shock. The goal alley used for one trial served as the start alley in the next trial. Throughout the experiment, the amplitude of the electrical shock-the minimum that would elicit a rapid response (intensity between .3 and $.5 \mathrm{~mA}$-was adjusted for each animal. The sequence of correct right $(\mathrm{R})$ and left $(\mathrm{L})$ turns in the Y-maze was determined according to the following pseudorandom schedule: L-L-R-L-R-R-L-R-R-L-R-L-L-R-R. Response latencies and number of errors were recorded.

After completion of the 15 training trials, the rats were removed from the maze and returned to their home cages, where they remained for varying intervals of time before being tested. As in Huppert and Deutsch's (1969) study, testing consisted of a retraining phase with a procedure identical to that of training, except that the animals were trained to a criterion of 10 consecutive correct responses. Using the paradigm adopted by Huppert and Deutsch, 9 animals that had attained the criterion during the training phase were discarded. When the criterion was not attained within 70 trials, the animals received a score of 80 . Response latencies and number of errors were also recorded during testing.

The experiment was run in three consecutive replications, and eight different retention intervals were studied: $5 \mathrm{~min}(n=14), 1 \mathrm{~h}$ $(n=15), 1$ day $(n=17)$, and $5(n=17), 8(n=16), 14(n=14), 21$ $(n=14)$, and $28(n=14)$ days. Each animal was assigned to one of the experimental groups, each representing a different trainingto-test interval (TTI), on the basis of its performance during the training phase. Response latencies and number of errors were pooled in blocks of five trials for acquisition and retention scores. A logarithmic transformation was applied to each response latency for statistical analysis. Savings in retention performance, that is, the differences between the scores from the first five testing blocks and the scores from the last five training blocks, were determined for response latencies and for errors.

\section{Results}

\section{Initial Training}

Because no significant differences among the three separate replications could be detected at the end of training, either for response latencies $[F(2,118)=.91]$ or for errors $[F(2,118)=1.78]$ the data were pooled for subsequent analyses.

An analysis of variance (group $\times$ repetition) performed on the three blocks of the training period and among the eight groups submitted to different training-to-test intervals showed: (1) no difference between groups for response latencies $[F(7,113)=1.37]$ or for errors $[F(7,113)=1.19],(2)$ no significant effect of repetition on response latencies $[F(2,113)=2.10]$ or on errors $[F(2,113)=.92]$, indicating no significant acquisition of the task, and (3) no interaction between groups and repetitions for the two parameters $[F(14,113)<1$ in each case]. Furthermore, no difference between the eight groups could be detected during the last block of training trials, either on response latencies or on errors $[F(7,113)<1$ in each case $]$.

\section{Retention}

Within-group comparisons indicated that the animals showed an increase in response latency only for TTI equal 
or superior to 21 days $[21$ days, $F(1,113)=6.03$, $p=.027 ; 28$ days, $F(1,113)=3.00, p=.10)$.

An overall analysis of variance performed on savings in performance indicated that there was no significant effect of TTI conditions on response latencies $[F(7,113)=$ $1.14]$ or on error scores $[F(7,113)=.69]$.

An overall analysis of variance performed on the mean number of trials to criterion as a function of the length of the TTI (see Figure 1) indicated a significant main effect of TTI conditions $[F(7,113)=5.49, p<.001]$.

Analyses of variance of paired comparisons indicated that the group of rats tested $1 \mathrm{~h}$ after training exhibited impaired performance relative to each of the remaining groups, except for the 28-day-TTI group ( $p<.001$ to $p=.049$ ). The 8- and 14-day-TTI groups [which did not differ from each other; $F(1,28)=.23$ ] were significantly better than any of the other groups $(p<.001$ to $p=.049$ ). The 28 -day-TTI group was slightly worse than the other groups, but the differences fell just short of significance [except when compared with the 8- and 14-dayTTI groups, which suggests a partial forgetting of initial training $(p=.085$ to $p=.13)]$.

\section{Discussion}

These results confirm that following partial brightnessdiscrimination escape training, reaching criterion can be transitorily facilitated following a TTI of several days (Huppert \& Deutsch, 1969). In addition, these data indicate that reaching criterion can also be transitorily disrupted after a TTI of $1 \mathrm{~h}$. This extends previous findings that showed similar results following either forced trials (Bryan \& Spear, 1976) or avoidance training (Barrett et al., 1971; Steranka \& Barrett, 1973). Finally, our data confirm that initial training is partially forgotten after an extended TTI (Huppert \& Deutsch, 1969).

The main point of interest in these results lies in the fact that the fluctuations of retention performance that correspond to the Kamin effect, to LTSI, and to spontaneous forgetting were all obtained after the same training episode. Thus, rather than being extrapolated from the results of different studies, the multiphasicity of the retention curve was actually demonstrated.

Nevertheless, the fact that there was no measurable evidence of original learning with either response latencies or errors raises a problem in the interpretation of these results. Obviously, the more probable explanation would be that some learning did occur during initial training, but that performance did not reflect it. Nevertheless, it might also be argued that poor or good test performance is due to nonassociative consequences of the electrical shocks delivered during the training episode rather than to variations in the strength of the memory trace resulting from previously acquired information. However, the fact remains that these fluctuations of performance are obtained only with the use of a criterion, the reaching of which implicates elaborative and integrative processes. They are not obtained with measures, such as response latency, that are more likely to be affected by the nonassociative effects of electrical shocks. This argues against the nonassociative interpretation.

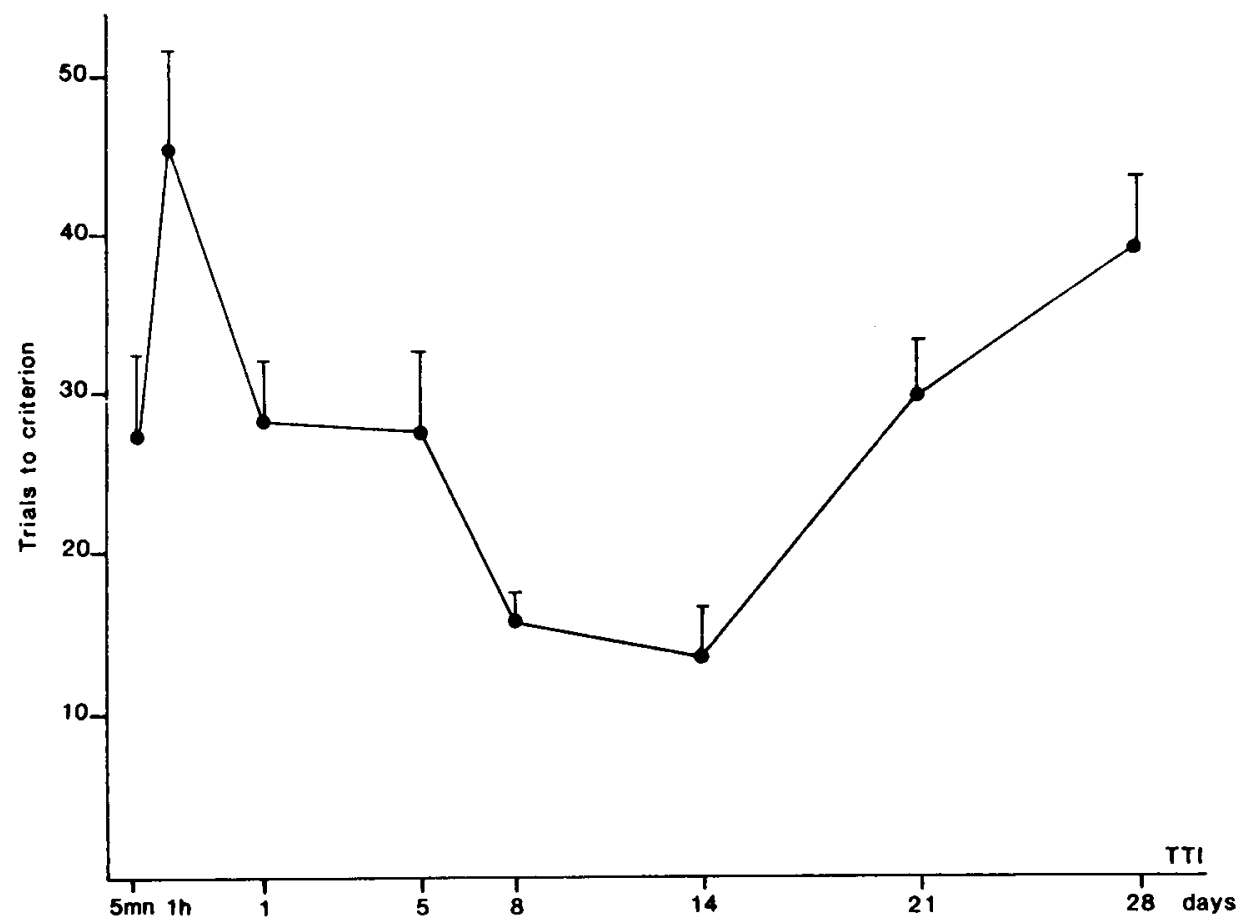

Figure 1. Mean number of trials $( \pm S E M$ ) needed to reach the test criterion of 10 consecutive correct responses as a function of the training-to-test interval (TTI). Note the spontaneous decrease in performance for the 1-h TTI group and the spontaneous increase in performance for the 8-and 14-day-TTI groups. 
Yet, use of the criterion of performance, which was adopted in this experiment to replicate the previous findings of Huppert and Deutsch (1969), gives rise to another problem. As a matter of fact, an alteration of this measure of performance, which is used only during testing and which implicates a large number of trials, could reflect a modification in acquisition capabilities rather than the retention level of initial training. To solve that problem conclusively, it would be useful to consider another discrete measure of performance, such as the score of an avoidance response, which can be studied during both phases of the experiment and thus permits a comparison to be made between terminal performance in acquisition and the first subsequent retention scores. Such an avoidance response score was, accordingly, used in Experiment 2.

\section{EXPERIMENT 2}

The first experiment confirmed that partial brightnessdiscrimination escape training yields long-term spontaneous improvement of performance (Huppert \& Deutsch, 1969) as well as a short-term performance decrement (the Kamin effect). The aim of the second experiment was to extend these results and to investigate the possibility that an active avoidance situation that leads to a Kamin effect (Barrett et al., 1971) might also yield a LTSI phenomenon. In addition, with an avoidance paradigm, an acquisition process that was not manifest in the first experiment could be expected, since it has already been demonstrated that extending the delay between a conditioned stimulus (CS) and an unconditioned stimulus (UCS) induces better training performance (N. H. Anderson, 1969). Furthermore, the avoidance response, which can be scored during both training and testing, might be susceptible to fluctuations as a function of the length of the retention interval.

\section{Method}

Subjects

The subjects were 105 male albino rats, with the same characteristics and housed in the same conditions as the rats in Experiment 1.

\section{Apparatus and Procedure}

All rats received pretraining and training according to the procedure of Experiment 1 . The only difference was that there was a 5 -sec interval between the CS and the UCS. When a rat entered the lighted arm within $5 \mathrm{sec}$, it successfully avoided shock; the score for this avoidance response was 2 . When the animal left the initial arm within $5 \mathrm{sec}$, its response was considered as a real attempt to avoid the shock and the score was 1 . All other responses, with the animals moving only after the onset of the shock, were termed escape responses.

During testing, the animals were retrained for 25 trials with the following sequence: L-R-R-R-L-L-R-L-L-L-R-L-L-R-L-R-R-R-LL-R-R-L-R-R. Three different measures of performance were recorded in the same manner as in Experiment 1: response latencies, number of errors, and number of avoidance responses.
Six animals were discarded because of their high response latencies $(>30 \mathrm{sec})$ in the last block of training trials.

At the end of training, the animals were returned to their home cages and then divided into six groups on the basis of their performance during the training phase. Six TTls were investigated: $10 \mathrm{~min}$ $(n=15), 1 \mathrm{~h}(n=18), 1$ day $(n=15)$, and $3(n=18), 5(n=15)$ and 21 days $(n=18)$. Because earlier studies had indicated that improvement occurs sooner with such an avoidance paradigm than with the escape paradigm of Experiment 1 (Gisquet-Verrier \& Alexinsky, 1983), the 8- and 14-day-TTI groups were omitted and a 5-dayTTI group was added.

\section{Results}

\section{Initial Training}

Analyses of variance (group $\times$ repetition) performed over the 15 training trials and among the six groups submitted to different TTIs indicated: (1) no difference between groups on response latencies $[F(5,93)=1.81]$, on errors $[F(5,93)=1.80]$, or on avoidance responses $[F(5,93)=.32] ;(2)$ a significant effect of repetition on response latencies $[F(2,93)=85.32, p<.001]$, on errors $[F(2,93)=22.56, p<.001]$, and on avoidance responses $[F(2,93)=18.31, p<.001]$; and $(3)$ no interaction between groups and repetitions $[F(10,93)<1$ in each case]. These analyses indicated acquisition during initial training for each of the three measures of performance considered.

\section{Retention}

Within-group comparisons. Animals assigned to a TTI of $10 \mathrm{~min}, 1$ day, or 5 days showed no alteration of performance between the last block of training trials and the first block of testing trials. Animals of the 3-day-TTI group exhibited a spontaneous improvement of performance as measured by their avoidance responses $[F(1,17)$ $=5.13, p=.035 \mathrm{~J}$. On the other hand, a decrement in performance was observed for a retention interval of $1 \mathrm{~h}$ for response latencies and for avoidance $[F(1,17)=8.86$, $p=.008$, and $F(1,17)=4.51, p=.046$, respectively]. Such a decrement was also observed at 21 days for response latencies and for avoidance responses $[F(1,17)$ $=4.31, p=.051$, and $F(1,17)=3.54, p=.074$, respectively].

Between-group analyses. An overall analysis of variance indicated a significant effect of the length of the TTI on the savings in number of avoidances $[F(5,93)=2.52$, $p=.034$ ] (see Figure 2). The same result was obtained for savings measured by response latencies $[F(5,93)=$ $2.29, p=.05]$, but not for savings in number of errors $[F(5,93)=.42]$.

Results of specific comparisons indicated that animals tested $1 \mathrm{~h}$ following training exhibited significantly less savings in performance than did those of the 1-day-TTI group $[F(1,31)=4.14, p=.047]$ or those of the 3-dayTTI group $[F(1,34)=9.40, p=.004]$ for avoidance responses, or than did those of the 10 -min-TTI group $[F(1,31)=7.03, p=.012]$ for response latencies. The 21 day-TTI group was also significantly disrupted relative 


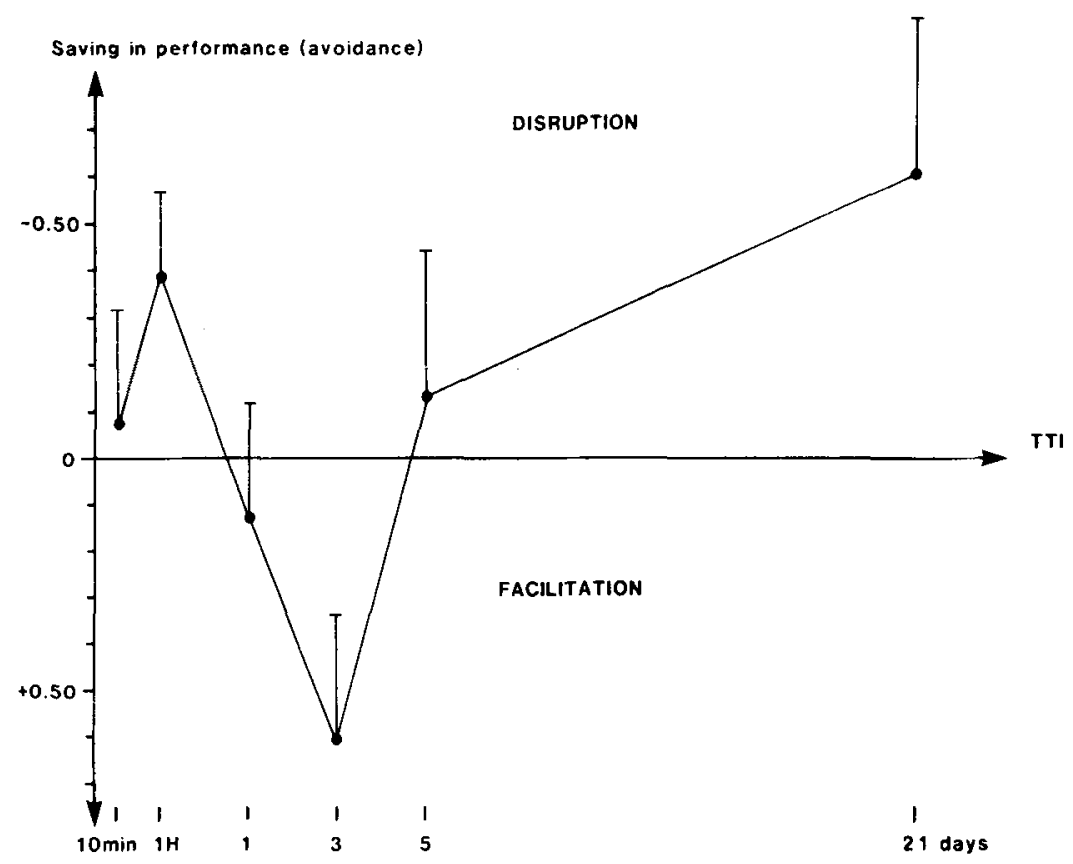

Figure 2. Mean score ( $\pm S E M$ ) for savings in number of avoidance responses corresponding to the difference in the number of avoidance responses between the first five test trials and the last five training trials, as a function of the training-to-test interval (TTI). Note the disruption in the avoidance score for the 1-H-TTI group and the improvement in performance for the 3day-TTI group relative to the level of performance at the end of training ( 0 line).

to the 1-day-TTI group for avoidance scores $[F(1,31)=$ $4.19, p=.045]$ and to the 3-day-TTI group for both avoidance responses $[F(1,34)=8.38, p=.006]$ and response latencies $[F(1,34)=5.23, p=.026]$. On the other hand, the 3-day-TTI group exhibited significantly more savings in number of avoidances than did any of the remaining groups [3-day/10-min, $F(1,31)=4.31$, $p=.042 ; 3$-day $/ 1$-day, $F(1,31)=3.95, p=.053 ; 3$-day $/ 5$ day, $F(1,31)=4.07, p=.049 ; 3$-day $/ 21$-day, $F(1,34)=$ 8.38, $p=.006]$, and significantly more savings on response latencies than did the $1-\mathrm{h}$-, the 3 -day- $[F(1,31)$
$=4.33, p=.043]$, or the 21-day-TTI groups $[F(1,34)=$ $5.23, p=.026]$. No other paired comparisons were significant.

Since the testing phase was limited to 25 trials in Experiment 2, the data obtained in both experiments cannot be shown in the same form. We did, however, compare the percentages of animals that reached the criterion of 10 consecutive correct responses within the first 25 testing trials in both experiments (see Figure 3). In the first experiment, this percentage fluctuated significantly over time $\left[\chi^{2}(7)=19.38, p<.01\right]$ much as did the number

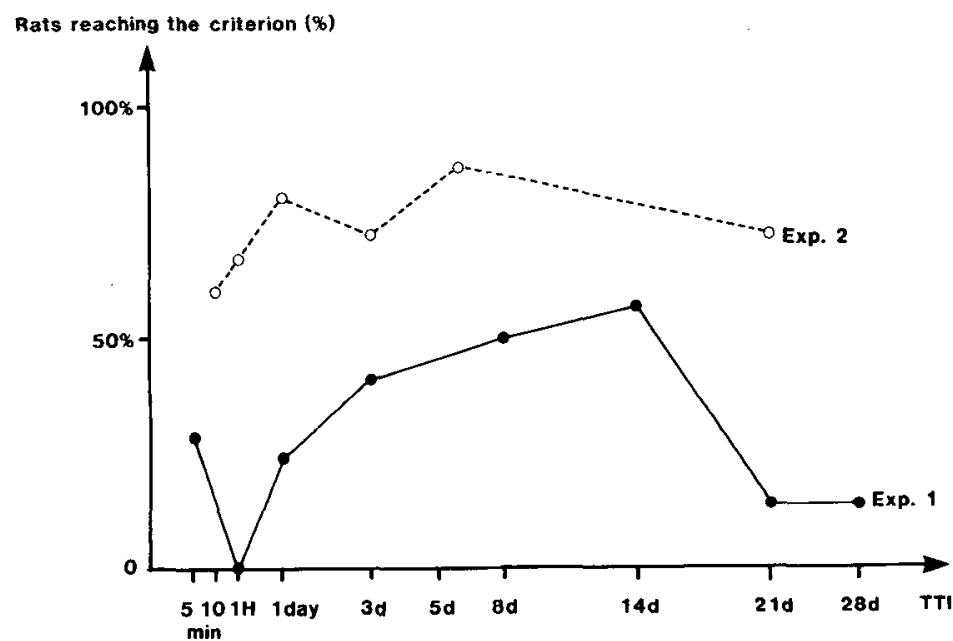

Figure 3. The percentages of animals reaching the criterion of 10 consecutive correct responses within the first 25 test trials for both experiments. Note that this percentage fluctuates in Experiment 1, whereas no modulation was obtained in Experiment 2. 
of trials to criterion in the overall testing session (see Figure 1). On the other hand, no variation was obtained on the same measure in Experiment $2\left[\chi^{2}(5)=3.42\right.$, n.s.]. These differences between the two experiments might be explained by the fact that only $29 \%$ of the animals reached criterion during the first 25 testing trials in Experiment 1, whereas $73 \%$ did so in Experiment 2. In other words, since most of the animals attained the criterion almost immediately in the avoidance situation, no modulation of this parameter could be expected. This observation provides further evidence of better acquisition of the light strategy in Experiment 2.

\section{Discussion}

The introduction of a 5-sec delay between CS and UCS, which provided an opportunity to avoid the footshock, had a twofold consequence: (1) It allowed us to obtain operational evidence of an acquisition process attested to by reductions in the response latencies and in the numbers of errors, as well as by an increase in the number of the avoidance responses, during the 15 training trials; and (2) it introduced a new measure-the elaborated avoidance response that can be studied during both training and testing.

The results of this experiment confirm those of the first experiment in showing a transitory decrease in performance (or Kamin effect) $1 \mathrm{~h}$ after initial acquisition, followed by spontaneous improvement of performance (LTSI), preceding the more durable decrement of performance resulting from spontaneous forgetting. Nevertheless, although the Kamin effect appears not to be affected by a modification of the training situation (avoidance vs. escape), the delay after which LTSI occurs is reduced.

It is worth noting, however, that the fluctuations of retention performance appear with some kinds of performance measures and not with others. Several explanations can be proposed to account for such discrepancies between the different indices of performance used in the two experiments.

The finding of no difference between groups in either experiment with respect to the rats' ability to correctly recall the safe stimulus confirms the findings of several authors (Barrett et al., 1971; Bryan \& Spear, 1976; Caul, Barrett, Thune, \& Osborne, 1974). In our specific situation, the number of errors committed by a rat was always very low, especially during testing. A study of the strategies used by the animal during acquisition of the rule indicates that, from the beginning of training, the animals responded according to various strategies, such as alternation (R-L-R-L), double alternation (R-R-L-L), or perseveration (R-R-R) (unpublished data). Acquisition of the light-discrimination response results from a comparison of the relative effectiveness of all these various strategies. Therefore, the number of "correct" responses due to the choice of these strategies must be added to the $50 \%$ chance of making a "correct" response. This explains the small number of errors made during training and supports the notion that the number of incorrect choices might not be a good index of learning for this task. To explain the failure to obtain a Kamin effect on choice behavior, Bryan and Spear (1976) emphasized that "choice response learning occurs more rapidly than avoidance response learning, leaving open the possibility that the choice response may be overlearned and rendered less sensitive to the effects of whatever mechanism is responsible for the phenomenon"' (p. 222). Such a position can also easily explain the absence of modulation in the number of animals reaching the criterion of performance in the present experiment (see Figure 3) as being due to a ceiling effect. Indeed, Huppert and Deutsch (1969) pointed out that after a training episode with a criterion of 10 consecutive correct responses, "the performance tends to be perfect during the retest, at least for the first 2 or 3 weeks after training"' (p. 267). Therefore, such a criterion can be considered as a good index of retention only after partial acquistion of the association rule, such as that found in Experiment 1.

The response latency measures the time needed to reach the lighted arm. Despite a logarithmic transformation, this response appears to be much more affected by poor performance (since the maximal total running time is $120 \mathrm{sec}$ ) than by good performance that leads to an avoidance response. Thus, this measure is more likely to reveal a decrement than an increment in performance and has often been considered as a demonstration of the Kamin effect. As illustrated by our own data, the Kamin effect can be assessed by an increase in the response latency, whereas LTSI cannot. The fact that an avoidance response only slightly affects a response latency score explains why, although these two indexes are obviously related, they must actually be considered as two different measures of performance.

The avoidance response is an integrated response that indicates not only a correct discrimination but also an understanding that shock can be prevented by making a rapid response. Nevertheless, a real avoidance response is difficult to obtain with a 5-sec delay between CS and UCS and within only 15 training trials. Therefore, we added to the "avoidance score" the attempts to avoid, which correspond to departures from the start arm within $5 \mathrm{sec}$ since those attempts constitute the first stage of the avoidance response. Under that condition, the number of avoidance responses reached a sufficient level during training to either increase or decrease during testing. Thus, savings in avoidance performance appears to be the best index for revealing fluctuations in retention performance in Experiment 2.

\section{GENERAL DISCUSSION}

These experiments confirm that retention performance following partial training of an aversively motivated brightness discrimination does not decrease over time according to a nonmonotonic function. Two transitory fluctuations of performance, the Kamin effect and long-term spontaneous improvement, as well as the long-lasting de- 
cay that corresponds to spontaneous forgetting, were obtained within the same experiment. These results were obtained repeatedly following 15 training trials, either in an escape situation, in which no acquisition was manifest, or in an avoidance situation, in which clear acquisition was observed. Thus, our results demonstrate the replicability of these fluctuations of retention performance and support the multiphasicity of the retention curve as well.

A comparison of the results obtained in both experiments indicates that the general shape of the retention curve does not seem to be dramatically affected by the training conditions, but that LTSI occurs sooner with the paradigm in which the acquisition process is operationally evidenced ( 3 days for the avoidance situation instead of 8 to 14 days for the escape situation). We made the same observation in an experiment in which we used an escape paradigm with two different amounts of initial training (15 or 25 trials) (Gisquet-Verrier \& Alexinsky, 1983). In that situation, LTSI also appeared sooner in the condition that led to the best acquisition ( 25 trials). Stanes et al. (1976) reported similar shifts in length of delay leading to LTSI, with animals divided into slow and fast learners, following an appetitive brightness discrimination. All of these results seem to suggest that the strengthening of initial training could reduce the delay after which spontaneous improvement occurs.

All of the methodological considerations developed in the discussion of Experiment 2 indicate that the choice of the measure of performance appears to be of critical importance to the demonstration of retention performance fluctuations. In fact, it appears that modulations of retention performance are best expressed when performance is assessed through rather complex measures. The best performance measure depends on the initial level of training (as it does for trials to criterion) and on the rature of the expected alterations (facilitation, disruption, or both).

Another point concerns what these performance fluctuations actually reflect. We have already noted that in most of the studies investigating LTSI, and especially the Kamin effect, retention performance is assessed on a relatively large number of test trials (50 for Barrett et al., 1971, and Steranka \& Barrett, 1973; approximately 40 in Experiment 1 and 30 for Huppert \& Deutsch, 1969, and Price \& Cooper, 1975; 25 for Bryan \& Spear, 1976). These results suggest that the two performance fluctuations can be due to greater or lesser capacity to solve, during testing, the problem raised by the experimental situation. However, the fact that significant differences between training and testing performance can be obtained suggests that, at least under our conditions, the Kamin effect corresponds to a real deterioration of performance, whereas LTSI reflects a real enhancement of performance between time of training and time of testing.

Two different hypotheses implicating either motivation or memory processing have been proposed to account for fluctuations in retention performance. According to an explanation in terms of motivational effect, the electrical shocks delivered during training induce an internal state that is responsible for poor performance (see Anisman, 1975) or for good performance (Price \& Cooper, 1975; Signorelli, 1976) during the retention test. The best argument for an explanation in terms of motivation has been found in the study of acquisition performance after varied intervals following a noncontingent footshock episode. Performance was shown to develop both according to an inverted-U-shaped curve that mimics the Kamin effect (Anisman \& Waller, 1971; Barrett et al., 1971) and according to a U-shape curve that mimics LTSI (Price \& Cooper, 1975). However, it must be noted that in most of these studies, the shock was delivered in the training apparatus, and associative processes highly relevant to the tested response, such as fear conditioned to the apparatus cues, could have occurred during the noncontingent footshock phase, even in the absence of explicit response training. As a matter of fact, it has been shown that when the environment in which noncontingent shocks are delivered is markedly different from the training environment, the previously evident U-shaped curves are not observed (de Toledo \& Black, 1970; Elson et al., 1977). This is the main reason why a noncontingent footshock control condition was not included in our experimental design. Another reason was that it had already been demonstrated that inescapable shocks produce interference with an organism's subsequent ability to learn a novel response to avoid or to escape shock (Maier \& Seligman, 1976). Because of this learned helplessness phenomenon, the noncontingent shock situation does not appear to be an appropriate condition for testing the nonassociative effects of footshocks. In our view, the best way to demonstrate a fluctuation of performance over time as being due to associative factors is to extend to another situation the results obtained initially.

The fact that the Kamin effect has been reported with an avoidance response measure (which might be readily affected by inhibitory processes) but not with a choice response measure (apparently a more accurate index of memory) has often been cited as perhaps the strongest objection to an interpretation of this phenomenon in terms of memory retrieval (Anisman, 1975; Steranka \& Barrett, 1973). However, our results indicated that the Kamin effect is obtained not only with avoidance responses but also with the use of a severe criterion of correct responses that appears to be much less affected by inhibitory processes. The absence of the Kamin effect on choice response seems to be due to the fact that the discrimination response is overlearned relative to the avoidance response (see discussion of Experiment 2). In addition, Klein and Spear (1970a) have demonstrated that animals do not seem to be inhibited $1 \mathrm{~h}$ after training, since they are not less able to cope with aversive conditioning. So, the Kamin effect could also correspond to more active responding at intermediate intervals (see also Bintz, Bradd, \& Brown, 1970; Singh, Sakellaris, \& Brush, 1971). On the other hand, it has been demonstrated that presentation of a training cue just prior to the retention 
test can abolish the transitory disruption of performance (Gisquet-Verrier, Dekeyne, \& Alexinsky, 1985; Klein \& Spear, 1970b). Thus, the Kamin effect appears to be mostly due to associative processes and to probably result from an internal state dissociation and/or to emotional factors that may interfere with the retrieval process.

Concerning LTSI, it is important to note that it has frequently been described following appetitive training to which an explanation in terms of shock reactivity is not relevant (Gisquet-Verrier \& Alexinsky, 1986; Jaffard et al., 1974; Stanes et al., 1976). We suggest, in agreement with others (Deutsch, 1971; Jaffard et al., 1974), that LTSI reflects an increase in memory strength over a period of several days. In the second experiment, the fact that a real improvement in performance was obtained between the end of training and the beginning of retraining supports such an interpretation. Moreover, it has been proposed that spontaneous improvement results from a consolidation process that may not be viewed as a simple phase of storage, but rather as an elaborative dynamic process (Jaffard et al., 1974). It has also been proposed that LTSI results from the dissipation of negative factors that built up during training and that interfere with the expression of what is really acquired at the time of training (Hull, 1963; McGeoch \& Irion, 1952). The present study does not allow us to make a decision on that particular problem.

The major point of this study is the demonstration that the Kamin effect and LTSI can be obtained consecutively following a training episode. Both are revealed either with an escape paradigm after 15 (Experiment 1) or 25 (Gisquet-Verrier \& Alexinsky, 1983) training trials, or with an avoidance paradigm (Experiment 2; GisquetVerrier et al., 1985). We have provided evidence that both the Kamin effect and LTSI result from associative processes. The fact that, in addition, these performance fluctuations have been consistently obtained following various training situations strengthens the notion that they must be considered as two regular and systematic features of the retention curve, at least following an aversive discrimination task.

We postulate that the multiphasic nature of the memory trace, which has been demonstrated by these experiments, might result from modulation of retrievability. Along with Underwood (1969) and Spear (1978), we consider that memory may be viewed as a collection of attributes, each representing events or features of the training episode, and that these attributes could develop over time rather independently. Thus, the systematic fluctuations of performance might be due to a reorganization among the different memory attributes. The Kamin effect and LTSI would then reveal two phases of this dynamic reorganization. To what extent this reorganization is spontaneous and to what extent it can be influenced by intervening events (such as pretest cuing) is the subject of our ongoing experimental work.

\section{REFERENCES}

ANDERSON, A. C. (1940). Evidence of reminiscence in the rat in maze learning. Journal of Comparative \& Physiological Psychology, 30, 399-411.

ANDERSON, N. H. (1969). Variation of CS-US interval in long-term avoidance conditioning in the rat with wheel turn and with shuttle tasks. Journal of Comparative \& Physiological Psychology, 68, 100-106.

ANISMAN, H. (1975). Time-dependent variations in aversively motivated behaviors: Nonassociative effects of cholinergic and catecholaminergic activity. Psychological Review, 82, 359-385.

ANisman, H., \& WALLER, T. C. (1971). Effects of conflicting response requirements and shock compartment confinement on the Kamin effect in rats. Journal of Comparative \& Physiological Psychology, 77, 240-244.

Ballard, P. B. (1913). Oblivescence and reminiscence. British Journal of Psychology, Monograph, 1, No. 2.

Barrett, R. J., Leith, N. J., \& RAY, C. S. (1971). Kamin effect in rats: Index of memory or shock-induced inhibition? Journal of Comparative \& Physiological Psychology, 77, 234-239.

Bintz, J., Bradd, W. C., \& Brown, J. S. (1970). An analysis of the role of fear in the Kamin effect. Learning \& Motivation, 1, 170-176.

Bovet, D., Bovet-Nitti, F. \& Oliverio, A. (1969). Genetic aspects of learning and memory in mice. Science, 163, 139-149.

BRUSH, F. R. (1971). Retention of aversively motivated behavior. In F. R. Brush (Ed.), Aversive conditioning and learning. (pp. 401-465). New York: Academic Press.

Bryan, R. C., \& SPEAR, N. E. (1976). A forgetting of a discrimination after intervals of intermediate length: The Kamin effect with choice behavior. Journal of Experimental Psychology: Animal Behavior Processes, 2, 221-234.

Caul, W. F., Barrett, R. J., Thune, G. E., \& Osborne, G. L. (1974). Avoidance decrement as a function of training-test interval: Single cycle or multiphasic? Behavioral Biology, 11, 409-413.

CHERKIN, A. (1971). Biphasic time course of performance after onetrial avoidance training in the chick. Communications in Behavioral Biology, 5, 379-381.

DE TOLEDO, L., \& BlaCK, A. H. (1970). Retention of aversively motivated responses in rats. Journal of Comparative \& Physiological Psychology, 71, 276-282.

DeuTsCH, J. A. (1971). The cholinergic synapse and the site of memory. Science, 174, 788-794.

Ebringhaus, H. (1885). Über das Gedächtnis: Untersuchen zur experimentellen Psychologie. Leipzig: Dunker \& Humbult.

Elson, I. J., SeyberT, J. A., \& Ghiselli, W. B. (1977). Retention of aversively motivated behavior: Effects of time of training and associative versus nonassociative processes. Behavioral Biology, 20, 337-353.

Geller, A., JARVIK, M. E. (1970). The role of consolidation in memory. In R. D. Bowman \& S. P. Datta (Eds.) Biochemistry in brain and behavior (pp. 125-137). New York: Plenum Press.

GisqueT-Verrier, P. (1983). L'effet Kamin: Description, analyse et interprétations. Revue critique. Cahiers de Psychologie Cognitive, 3 , 3-24.

Gisquet-Verrier, P., \& AleXinsky, T. (1983). Modulation of longterm memory evolution by pretest cueing in the rat. Behavioral Brain Research, 8, 249.

GISQUET-VERRIER, P., \& ALEXINSKY, T. (1986). Does contextual change determine long-term forgetting? Animal Learning \& Behavior, 14, 349-358.

Gisquet-VERrier, P., Dekeyne, A., \& Alexinsky, T. (1985). Memory reorganization over time as revealed by interaction between type of pre-test cueing and length of retention interval. In B. E. Will, P. Schmitt, \& J. C. Dalrymple-Alford (Eds.), Brain plasticity, learning and memory (p. 561). New York: Plenum Press.

Holloway, F. A., WANSLEy, R. A. (1973). Multiple retention deficits at periodic intervals after passive avoidance learning. Science, 80, 208-210. 
Hull, C. L. (1963). Principles of behavior. New York: AppletonCentury-Crofts.

Hunsicker, J. P., \& Mellgren, R. L. (1977). Multiple retention deficits in the retention of an appetitively motivated behavior across a 24-h period in rats. Animal Learning \& Behavior, 5, 14-16.

HuPPERT, F. A., \& DeUTSCH, J. A. (1969). Improvement in memory with time. Quarterly Journal of Experimental Psychology, 21, 267-271.

Jaffard, R., Destrade, C., Soumireu-Mourat, B., \& Cardo, B. (1974). Time-dependent improvement of performance on appetitive tasks in mice. Behavioral Biology, 11, 89-100.

Kamin, L. J. (1957). The retention of an incompletely learned avoidance response. Journal of Comparative \& Physiological Psychology, 50, $457-460$.

KLEIN, S. B., \& SPEAR, N. E. (1970a). Forgetting by the rat after intermediate intervals ("Kamin effect") as retrieval failure. Journal of Comparative \& Physiological Psychology, 71, 165-170.

KLEIN, S. B., \& SpeAR, N. E. (1970b). Reactivation of avoidancelearning memory in the rat after intermediate retention intervals. Journal of Comparative \& Physiological Psychology, 72, 498-504.

MAGDsicK, W. K. (1936). The curve of retention of an incompletely learned problem in albino rats at various age levels. Journal of Psychology, 2, 25-48.

Maier, S. F., \& Seligman, M. E. P. (1976). Learned helplessness: Theory and evidence. Joumal of Experimental Psychology: General, $105,3-46$.

MCGEOCH, J. A., \& IRION, A. L. (1952). The psychology of human learning. New York: Longmans Green.

Messenger, J. B. (1971). Two stage recovery in Sepia. Nature, 232, 202-203.

Pinel, J. P. S., \& CoOper, R. M. (1966). Demonstration of the Ka- min effect after one-trial avoidance learning. Psychonomic Science, 4, 17-18.

Price, M. T. C., \& CoOper, R. M. (1975). U-shaped functions in a shock-escape task. Journal of Comparative \& Physiological Psychology, 89, 600-606.

SANDERS, G. D. \& BARLow, J. J. (1971). Variations in retention performance during long-term formation. Nature, 232, 203-204.

SiGNORELLI, A. (1976). Influence of physostigmine upon consolidation of memory in mice. Journal of Comparative \& Physiological Psychology, 90, 658-664.

Singh, P. J., Sakellaris, P. C., \& Brush, F. R. (1971). Retention of active and passive avoidance responses tested in extinction. Leaming \& Motivation, 2, 305-323.

SPEAR, N. E. (1978). The processing of memories: Forgetting and retention. Hillsdale, NJ: Erlbaum.

Stanes, M. D., Brown, C. P., \& Singer, G. (1976). Effect of physostigmine on Y-maze discrimination retention in the rat. Psychopharmacologia, 46, 269-276.

Steranka, L. R., \& Barrett, R. J. (1973). Kamin effect in rats: Differential retention or differential acquisition of an active avoidance response? Journal of Comparative \& Physiological Psychology, 85, 324-330.

Underwood, B. J. (1969). Attributes of memory. Psychological Review, 76, 559-573.

Wansley, R. A., Holloway, F. A. (1975). Multiple retention deficits following one-trial appetitive training. Behavioral Biology, 14, 135-149.

(Manuscript received November 18, 1986; revision accepted for publication May $11,1987$. ) 